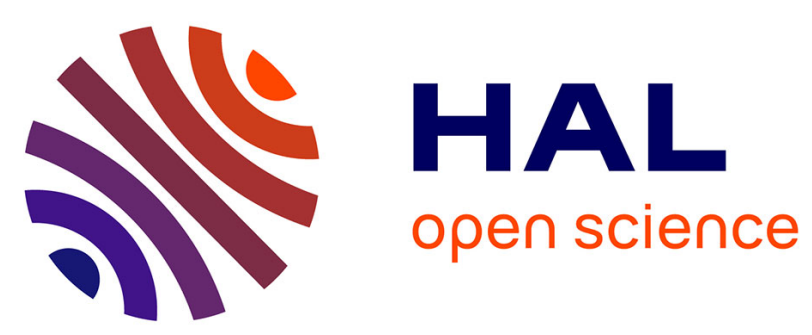

\title{
Les publics des tauromachies
}

Catherine Bernié-Boissard

\section{To cite this version:}

Catherine Bernié-Boissard. Les publics des tauromachies. DU TAUREAU ET DE LA TAUROMACHIE Hier et aujourd'hui , , p. 43-53, 2012, DU TAUREAU ET DE LA TAUROMACHIE Hier et aujourd'hui, 9782354122751. 10.4000/books.pupvd.3154 . halshs-01635164

\section{HAL Id: halshs-01635164 https://shs.hal.science/halshs-01635164}

Submitted on 20 Nov 2017

HAL is a multi-disciplinary open access archive for the deposit and dissemination of scientific research documents, whether they are published or not. The documents may come from teaching and research institutions in France or abroad, or from public or private research centers.
L'archive ouverte pluridisciplinaire HAL, est destinée au dépôt et à la diffusion de documents scientifiques de niveau recherche, publiés ou non, émanant des établissements d'enseignement et de recherche français ou étrangers, des laboratoires publics ou privés. 


\section{Les publics des tauromachies}

Catherine Bernié-Boissard

Attaché à la figure du taureau, ou séduit par le combat entre l'homme et l'anomal, le public des corridas et celui des courses libres, en Languedoc, est à la fois très contrasté du point de vue sociologique et très proche du point de vue culturel. C'est la conclusion qui peut être tirée de deux enquêtes réalisées au milieu de la décennie : la première en 2004 lors de la feria de Pentecôte à Nîmes, auprès des spectateurs de la corrida - course à l'espagnole avec mise à mort ; la seconde, la même année, dans les arènes de la bouvine - course camarguaise sans mise à mort, localisées dans les petites et moyennes communes de la région Languedoc-Roussillon.

Pour différentes raisons, tenant notamment à la présence du fait tauromachique à l'échelon régional, un projet d'enquête s'est en effet noué, en 2004, à l'université de Nîmes, autour de l'élaboration et de la passation d'un questionnaire dont les réponses, collectées à l'entrée des arènes de la ville auprès de 418 spectateurs des corridas de la Féria de Pentecôte, permettent de juger des connaissances d'un public précis en matière tauromachique, mais aussi de sa composition sociale, de son mode de fréquentation des spectacles taurins, de ses origines démographiques ou géographiques, de son engagement dans d'autres types de pratiques collectives culturelles ou sportivesi.

La publication des résultats, mettant en évidence le caractère endogène du renouvellement du public, peu ouvert hors les cercles d'initiés, suscitèrent l'intérêt des reponsables de la Fédération de la course camarguaise. Dans le but de mieux connaître les amateurs de la course, un questionnaire fut proposé à 300 spectateurs, les jours de manifestations, dans les arènes de la région ${ }^{i i}$. L'analyse de ces deux études exploratoires permet ainsi de cerner quelques aspects du profil à la fois proche et contrasté de ces publics.

Le public de la corrida est plutôt urbain, diplômé, il dispose de revenus élevés, tandis celui de la course camarguaise est plus rural, moins diplômé et dispose de revenus plus modestes. Dans les deux cas cependant, il apparaît que la reproduction s'effectue essentiellement de manière endogène, par la famille ou le cercle d'amis proche. Le caractère festif de la feria, dont la figure du taureau est devenue prétexte, favorise l'élargissement du public de la corrida, tout comme les évènements organisés autour de la course libre, sans toutefois assurer un renouvellement au delà des cercles traditionnels.

\section{Deux figures du spectateur}

Le profil du spectateur de la corrida à l'espagnole paraît simple à dégager: l'aficionado aime le spectacle tauromachique. Il revient souvent, ici ou ailleurs. C'est une figure plutôt masculine, urbaine, disposant de revenus assez élevés. C'est un multi-appartenant qui évolue au sein de cercles de socialisation diversifiés (clubs taurins, culturels, sportifs). Mais il ne s'agit là que de l'une des figures - la plus nettement marquée - d'un ou plutôt des publics, composés d'anciens et de nouveaux, connaisseurs ou néophytes, "pratiquants » ou simples fidèles, mais aussi de curieux amenés là par les hasards de la fête. Avec ses rites, ses complicités d'initiés, la société tauromachique prône des vertus machistes, comme l'écrit Michel del Castillo: le courage, une virilité hautaine et dure ..., mais elle ne se résume pas à cela. 
Pour la course camarguaise, le public est majoritairement masculin (dans l'échantillon étudié), essentiellement âgé de plus de 35 ans et plutôt rural, il comporte de nombreux retraités.

On verra que la corrida et la course camarguaise forment un monde commun mais différencié par ses appartenances sociales. La question posée à l'issue des deux enquêtes est celle $d u$ renouvellement d'un public dont les formes d'intégration relèvent du cercle familial ou amical, au sein d'une aire culturelle dont les frontières ne bougent pas - le sud de la France, les villes et villages de tradition taurine, et dans un moment - les débuts du XXIè siècle, où le spectacle de la corrida avec mise à mort est ouvertement contesté.

\section{Une culture localisée}

La Féria est communément présentée comme l'une des plus grandes fêtes en Europe, attirant un large public venu de toute la France et des pays voisins. Le décalage est net avec la corrida, qui demeure pour l'essentiel un spectacle de gens du Sud, languedociens, habitants du sud-ouest où les traditions tauromachiques ont été maintenues, ou du sud-est, d'où elles ont aujourd'hui quasiment disparu.

Cette polarisation s'explique par l'histoire mouvementée de ce spectacle, autorisé seulement dans les villes de tradition. Ce sont donc bien ces villes qui fournissent les trois-quart du public, comme le confirment les études publiées par le gestionnaire des arènes à partir de la billetterie. Mieux encore, près de 6 spectateurs sur 10 sont originaires du Languedoc-Roussillon, étant donné l'attractivité de la Féria de VicFezensac à la même période pour le sud-ouest. L'enquête, sur ce point, confirme le caractère localisé de la culture taurine en Europe.

Un spectateur sur cinq vient donc des régions situées au-delà de la frontière culturelle d'Occitanie, avec une petite minorité parisienne et, par ailleurs, des origines très dispersées pour les étrangers: Londres, Florence, Maastricht ou Athènes, New-York ... et bien sûr l'Espagne.

Pour la course camarguaise, le public vient de l'immédiate proximité : communes moyennes ou petites pour l'essentiel des départements des Bouches du Rhône, du Gard et de l'Hérault. Les grandes agglomérations sont peu représentées. Il y a dans l'échantillon peu de personnes originaires de départements plus éloignés (quelques uns du Sud de la France), pas d'étrangers. .

\section{La corrida : un public élitiste}

L'aficionado, on l'a dit, est plutôt masculin : 6 hommes sur 10, pour 4 femmes, en général. Il se recrute plutôt dans la catégorie des actifs : les moins de 20 ans et les plus de 60 ans sont peu nombreux. L'essentiel est formé par des adultes entre 20 et 60 ans (8 sur 10$)$.

La figure de l'ouvrier, comme celle du chômeur, est rare (moins de $2 \%$ ). Celle de l'employé, des professions intermédiaires ou des cadres, disposant de revenus moyens ou élevés, en rapport avec le coût du spectacle, domine largement.

On observe donc une nette éviction des catégories populaires, puisqu'en Languedoc Roussillon par exemple les ouvriers forment le cinquième des actifs et plus encore au niveau national. A l'inverse, les cadres et professions intellectuelles 
supérieures doublent leur poids relatif dans la population, tandis qu'employés et professions intermédiaires sont sous-représentés. Décalage donc, là encore, entre spectacle populaire et public élitaire, entre le caractère festif et ouvert de la Féria et la relative fermeture des arènes.

Les aficionados représentent un auditoire sur diplômé, au-delà de Bac+2 (près de 4 sur 10 contre 1 en moyenne en France), tandis que les moins diplômés, avant le niveau du Bac, sont minoritaires (moins de 2 contre 6,5 en moyenne).

Le public, toutes proportions gardées, présenterait alors des similitudes avec celui de l'Opéra, qui, spontanément, attire les plus diplômés et les plus favorisés du point de vue socioprofessionnel.

Toutefois, la corrida ne présente pas en tant que spectacle le caractère élitiste de l'Opéra. C'est donc moins dans le rapport au spectacle qu'il faut chercher l'explication de l'effacement des couches populaires, que dans la question de l'accès au spectacle lui-même, autrement dit sa démocratisation, au sens le plus élémentaire du terme. La distribution est meilleure pour le type d'études, avec une légère majorité des scientifiques par rapport aux littéraires ou à ceux qui ont fait des études techniques ou générales.

\section{Course camarguaise : un public populaire}

La démographie rejoint le comportement: au nombre élevé de personnes retraitées ou proches de la retraite correspond un public ancien, assidu, peu marqué par le renouvellement. Il faut souligner le caractère populaire du public, très différent de celui de la corrida. En dehors des retraités, il y a une majorité d'employés, ouvriers et de professions intermédiaires salariées, peu de cadres ou chefs d'entreprise. Les fonctionnaires sont bien représentés, notamment chez les fonctionnaires territoriaux.

Plus de la moitié de la population étudiée a un faible niveau d'études, ce qui s'explique sans doute par l'âge. Les plus jeunes sont aussi plus diplômés. Il y a cependant une représentation de tous les niveaux d'études.

\section{Des publics ...}

L'enquête auprès du public de la corrida met en évidence deux figures du spectateur :

- d'une part celle du public régulier, représentant 6 spectateurs sur 10, qui fréquente les arènes depuis plus de 10 ans, à $80 \%$.

- d'autre part celle du public occasionnel, minoritaire, qui vient pour la première fois, ou seulement à la Féria, ou encore de manière intermittente.

La première accuse les traits décrits plus haut, tandis que la seconde est plus jeune, plus étudiante et légèrement plus populaire. On pourrait en tirer la conclusion que la reconduction est assurée par le glissement des nouveaux spectateurs vers le groupe des assidus. Or le processus de reproduction paraît plus complexe.

Un tiers des spectateurs réguliers sont des cadres ou professions libérales, majoritaires, avec les professions intermédiaires. Ils se distinguent par le diplôme : plus de 4 sur 10 est à $\mathrm{Bac}+4$, et au total 6 sur 10 à $\mathrm{Bac}+2$. 
Le public occasionnel, plus jeune, très diplômé, appartient aux catégories favorisées du point de vue scolaire et socioprofessionnel. Plus jeune et plus féminin que le premier, il paraît mieux refléter la sociologie de la population.

Ces résultats confirment la photographie d'un public quelque peu élitiste, plus scientifique pour les réguliers, plus littéraire pour les autres, loin de l'image d'un spectateur inculte, avide de sensations fortes. Toutefois, le diplôme donne rarement accès à une bonne connaissance de la tauromachie: les étudiants, les diplômés à $\mathrm{Bac}+2$, sont les moins compétents, alors que les meilleurs experts se trouvent chez les moins diplômés, ou encore les ouvriers.

Le public de la course camarguaise est également adepte de longue date. Plus de trois-quarts des spectateurs assistent régulièrement à la course. Une petite minorité vient moins de 5 fois/an ou pour la première fois.

Le public assidu est pour l'essentiel un public adulte de plus de 35 ans, retraité pour plus d'un tiers. Il est ancien, il assiste à la course depuis plus de 10 ans. Un spectateur sur dix vient depuis moins de 5 ans, le pourcentage étant plus faible pour les 5 à 10 ans, ce qui interroge là encore le renouvellement de ce public.

\section{Pratiques du spectacle : quels renouvellements?}

Les aficionados réguliers, les plus nombreux, sont aussi les plus anciens. Les occasionnels de la corrida se répartissent entre anciens et nouveaux, mais avec un pourcentage plus élevé de spectateurs de moins de 5 ans. Autrement dit, une majorité du public peut être considérée comme fidélisée. Une minorité, attirée par le spectacle, ne renouvelle pas systématiquement l'expérience, ce qui explique que les novices soient plus nombreux que les occasionnels: le néophyte est un spectateur incertain ...

La déperdition est de $30 \%$ environ entre le groupe des moins de 5 ans et celui des 5 à 10 ans. Pour assurer un renouvellement constant, il serait donc nécessaire de disposer d'une base plus large de nouveaux venus.

On vérifie que la fête favorise cet élargissement : le public qui vient seulement à la Féria est le seul où le poids des anciens diminue, au profit des spectateurs plus récents. Cependant, ce public là se déclare aussi comme le moins expert, ce qui, sans doute, ne favorise pas sa fidélisation. L'élargissement du public ne saurait donc s'effectuer sans transmission des règles de la culture tauromachique.

La problématique est à peu près identique pour le public de la course camarguaise, dont la connaissance relève pour l'essentiel du cercle familial ou amical. Le public se renouvelle donc de manière endogène, dans une région très attractive où la population nouvellement installée ignore le monde de la «bouvine » et ses règles. Ces résultats interrogent la possibilité d'intégration d'un public nouveau, n'appartenant pas à la culture de la course.

\section{Intégrations au monde taurin}

Le spectacle de la corrida avec mise à mort n'est pas le moment privilégié de transmission des règles, ce qui semble confirmé par l'usage différent des autres types d'activités durant la Féria. Les plus assidus participent plus que la moyenne aux 
rencontres taurines mais également aux expositions, souvent liées à cette approche, tandis que les autres préfèrent le caractère festif des bodegas, des concerts ou de la rue.

Les pratiques se différencient encore par l'ouverture au monde des tauromachies. Ainsi les habitués sont trois fois plus nombreux à suivre des corridas à cheval, ils apprécient les courses camarguaises, tandis que l'intérêt décroît chez les néophytes. Cependant, la moitié du public connaît les courses camarguaises, ce qui confirme son enracinement régional. Par ailleurs, un grand nombre suit les corridas en France, mais l'intérêt pour le spectacle en Espagne progresse à mesure qu'augmente la passion.

C'est dans l'intégration à l'univers taurin que se trouve la ligne de partage la plus significative entre réguliers et occasionnels. Les habitués sont aussi majoritairement membres des clubs taurins, plus particulièrement lorsqu'ils ont un faible niveau d'études. Ils sont cinéphiles, à proportion de 3 pour 10 environ, abonnés à des revues spécialisées. Les autres spectateurs ignorent les clubs, sont très peu abonnés à des revues et ne voient guère de documentaires.

L'écart est encore plus grand pour la littérature, appréciée par les réguliers, surtout les plus diplômés (6 sur 10) ou, curieusement, les militaires mais assez peu par les autres (1 sur 10). Littérature et cinéma sont les modes d'accès des plus diplômés. La télévision, en revanche, rassemble les publics, elle est regardée par 5 assidus sur 10 et plus de 4 occasionnels, notamment les moins diplômés.

Le milieu familial ou amical est essentiel à la découverte, la famille étant le mode d'accès privilégié des connaisseurs. Les moins experts sont plus souvent initiés par des amis et pour les plus récents par la télévision. Logiquement, les usages des assidus sont associés à une bonne, voire une excellente connaissance des règles. Leur forte intégration au monde taurin se vérifie encore par le fait de se déplacer avec un club, et d'avoir assisté à la première corrida de la Féria, celle du mercredi.

Le spécialiste, plutôt cadre, profession libérale, ou intermédiaire, voire militaire, est abonné, plutôt bien placé, par exemple au toril. Il est assidu, depuis plus d'une décennie. Membre d'un club taurin, il se déplace volontiers, jusqu'en Espagne. Il entretient ses connaissances grâce au cinéma, à la vidéo, la télévision, au livre ou aux revues spécialisées.

Les occasionnels, plutôt spectateurs du dimanche de la Féria, ont une faible connaissance des règles. Ils sont moins bien intégrés à cet univers. Le néophyte, plutôt jeune, étudiant ou employé, n'est pas abonné, car il s'agit d'un investissement risqué pour celui qui n'est pas sûr d'apprécier, il est placé en amphi. Il vient épisodiquement, depuis peu de temps. Il n'est pas adhérent d'un club taurin. Il se déplace parfois, à proximité, en France, pour assister à des corridas. Il lit peu de livres ou de revues spécialisées, regarde peu de films ou de documentaires, excepté à la télévision.

Enfin, un environnement familial et amical peu hostile à la corrida favorise l'intégration à son univers. L'hostilité de la famille est en effet croissante à mesure que décline la fréquence d'assistance. Elle est plus vive de la part des amis pour les nouveaux spectateurs. Etonnamment, l'hostilité des amis ou des collègues de travail est moins exacerbée vis-à-vis du public de la Féria, sans doute en raison de son appartenance à l'espace local, de tradition taurine. 
On observe cependant un taux significatif de non réponses, chez les assidus, qui témoigne d'un certain embarras à évoquer ces questions.

Les engagements des aficionados trouvent d'autres terrains d'exercice : la culture ou les sports les mobilisent, toutefois les plus jeunes sont moins investis dans la vie civique ou syndicale.

\section{Course camarguaise : du côté de la tradition ...}

Les groupes folkloriques et la corrida à l'espagnole attirent moins l'amateur de course libre que les fêtes, les spectacles de rue, les ferrades et les clubs. La tradition, les fêtes traditionnelles, rassemblent très largement le public, quelque soit son âge ou ses appartenances socioprofessionnelles.

Les termes pour désigner la course sont très variés, en tête viennent la tradition, la passion, la fe, l'admiration pour le duel entre l'homme et le taureau, le courage des raseteurs, le respect des hommes et des animaux, la beauté des rasets. A l'exception de quelques commentaires désenchantés, les jugements portés sur le spectacle sont positifs. La figure du taureau (ou des taureaux, du toro, du biou) domine les appréciations. Par ailleurs, ce sont les qualités morales attachées à la manifestation qui plaisent au public (honnêteté, courage, probité ...etc.). Il y a très peu de mentions négatives, toujours nuancées.

Une partie des spectateurs exprime nettement son refus de la mise à mort, donc de la corrida à l'espagnole, ce qui rejoint les nombreuses mentions sur le « respect du taureau » dans les appréciations de la course.

\section{Esthétique, peur délicieuse ...}

Pour terminer, il faut souligner quelques traits.

Le premier est celui du caractère plutôt érudit du public de la corrida, attaché à valoriser un patrimoine, transmettre des traditions, quelle que soit par ailleurs sa position sociale. Ce public est celui d'un évènement culturel, plutôt que sportif ou d'une autre nature.

Le deuxième est la permanence de l'attractivité des courses de taureaux, malgré le développement de mouvements hostiles, pour un public jeune, néophyte, fortement diplômé. Les termes utilisés pour caractériser la course à l'espagnole, comme de la cours camarguaise, relèvent en premier lieu du lexique de l'art, de la fête, de l'émotion, ils sont du registre de l'expérience esthétique.

Le troisième est l'enchevêtrement des clivages, sociaux, professionnels, taurins, sur les gradins de l'amphithéâtre, où les moins diplômés sont les mieux dotés en « capital tauromachique », tandis que l'élite intellectuelle vient y prendre des leçons. N'y a-t-il pas là une sorte de « revanche sociale »?

Cependant, ces remarques appellent quelques commentaires. Le spectacle tauromachique à Nîmes serait-il devenu un signe distinctif des classes supérieures, éliminant les classes populaires? Une sorte de «distinction», permettant l'expression des goûts et des affinités d'une partie des classes moyennes?

On peut, à la lecture des résultats de l'enquête, se demander si l'attractivité du spectacle permet la pérennité de l'intérêt des nouveaux spectateurs, sans que soient interrogés les modes d'accès, la composition sociale du public ... La fidélisation ne 
procède pas d'un mouvement mécanique, mais d'une transmission par la famille, les amis, les lieux de sociabilité, les médias ...

\footnotetext{
${ }^{\mathrm{i}}$ « Pratiques de la tauromachie : le public des arènes de Nîmes pendant la Féria de Pentecôte (26-30 mai 2004)», travail exploratoire visant à mieux connaître les pratiques individuelles liées à la tauromachie. Enquête conduite en partenariat avec le Cercle Universitaire d'Etude et de Médiation des Cultures et des Pratiques Taurines et une vingtaine d'étudiants du Centre Universitaire de Nîmes. Bernié-Boissard, C., Fournier, S.n (dir.), Tauromachies, sports, culture, Regards croisés sur les publics, L'Harmattan, 2006.

iiii «Enquête FFCC: le public des arènes de la course camarguaise, juin-septembre 2005 », travail exploratoire visant à mieux connaître le public de la «bouvine ». Questionnaire d'enquête réalisé à la demande de la FFCC, qui en a assuré la passation. Les résultats et les commentaires portent sur 150 réponses au questionnaire (la moitié du total). L'échantillon a été pris au hasard, il représente le public enquêté. Ces résultats ne prétendent pas refléter la réalité de tout le public de la course libre, car le document a été distribué/administré au public les jours de manifestations, sans aucun autre critère de sélection que la diversité des arènes, des jours et donc du public lui-même. Cette diversité autorise toutefois à considérer que cet échantillon est représentatif de la population qui assiste aux courses. Les résultats ne sont pas publiés, ils ont été diffusés par la FFCC auprès de ses adhérents.
} 